EXTENDED REPORT

\title{
Inverse association between circulating APRIL levels and serological and clinical disease activity in patients with systemic lupus erythematosus
}

\author{
W Stohl, S Metyas, S-M Tan, G S Cheema, B Oamar, V Roschke, Y Wu, K P Baker, D M Hilbert
}

Ann Rheum Dis 2004;63:1096-1103. doi: 10.1136/ard.2003.018663

See end of article for authors' affiliations .....................

Correspondence to: Dr W Stohl, Division of Rheumatology, University of Southern California, 2011 Zonal Avenue, HMR 711, Los Angeles, CA 90033, USA; stohl@ usc.edu

Accepted 2 February 2004

\begin{abstract}
Objective: To assess longitudinal expression of a proliferation-inducing ligand (APRIL) in patients with systemic lupus erythematosus (SLE) and its correlation with B lymphocyte stimulator (BLyS) expression, serum anti-dsDNA titres, and clinical disease activity.

Methods: Sixty eight patients with SLE were longitudinally followed up for a median of 369 days. At each visit the physician assessed disease activity by SLEDAl, and blood was collected for determination of serum APRIL and BLyS levels and of blood APRIL and BLyS mRNA levels. Fiffeen normal control subjects underwent similar laboratory evaluation.

Results: Dysregulation of APRIL was not as great as that of BLyS. Changes in serum levels of APRIL and BLyS over time were usually discordant, whereas blood levels of APRIL and BLyS mRNA strongly paralleled each other. Serum APRIL levels modestly, but significantly, inversely correlated with serum anti-dsDNA titres in anti-dsDNA positive patients analysed in aggregate. Moreover, serum APRIL levels modestly, but significantly, inversely correlated with clinical disease activity in all patients analysed in aggregate.

Conclusion: Serum levels of APRIL and BLyS are differentially regulated. APRIL may serve as a down modulator of serological and/or clinical autoimmunity in patients with SLE. This may have important ramifications for BLyS targeted treatment, and it remains to be determined whether agents which neutralise only BLyS will be preferable to agents which neutralise both BLyS and APRIL.
\end{abstract}

S everal members of the tumour necrosis factor (TNF) ligand superfamily have emerged as candidate targets of biological agents for the management of patients with systemic lupus erythematosus (SLE). One such TNF ligand superfamily member is B lymphocyte stimulator (BLyS; trademark of Human Genome Sciences, Inc (HGS), Rockville, MD; also known as BAFF, TALL-1, THANK, TNFSF13B, and zTNF4), ${ }^{1-6}$, a potent B cell survival factor. ${ }^{7-10}$

Substantial evidence has accumulated in support of BLyS as a therapeutic target in SLE. Constitutive overproduction of BLyS in some (albeit not all) mice transgenic for the human or murine blys gene leads to SLE-like features, including raised titres of multiple autoantibodies (including antidsDNA), circulating immune complexes, and renal immunoglobulin deposits. ${ }^{611} 12$ Of great importance, cross sectional and longitudinal studies have documented overexpression of BLyS in $>50 \%$ of human patients with $S L E,{ }^{13-15}$ pointing to a role for BLyS in human disease as well. Moreover, treatment of SLE prone $(\mathrm{NZB} \times \mathrm{NZW}) \mathrm{Fl}$ and MRL-lpr/lpr mice with a BLyS antagonist ameliorates progression of disease and improves survival. ${ }^{6}{ }^{16}$ Although the efficacy of BLyS antagonism in human patients with SLE remains to be formally proved, a fully human anti-BLyS monoclonal antibody ${ }^{17}$ has undergone phase I testing in patients with SLE and has been shown to be safe and biologically active. ${ }^{18} \mathrm{~A}$ phase II clinical trial with this anti-BLyS monoclonal antibody in SLE has recently begun.

Another member of the TNF ligand superfamily related to, but distinct from, BLyS is a proliferation-inducing ligand (APRIL, also known as TNFSF13A), a 250 amino acid protein that binds to two of the three BLyS receptors (BCMA and TACI $)^{19-23}$ but not to the third BLyS receptor, BAFFR. ${ }^{24}{ }^{25}$ The role, if any, for APRIL in SLE pathogenesis is uncertain. In contrast with the development of SLE-like features in blys transgenic mice, april transgenic mice (which constitutively overexpress APRIL) develop neither overt B cell abnormalities nor serological and/or clinical autoimmunity. ${ }^{26}$ Because APRIL does not bind to BAFFR, the receptor through which BLyS predominantly mediates its agonist effects on B cells, ${ }^{16} 242527$ it had been felt that APRIL was relatively inert in its effect on B cell biology. However, this belief needs to be modified, and APRIL may importantly contribute to B cell homoeostasis. On the one hand, APRIL and BLyS can each independently promote $\mathrm{T}$ cell independent class switching of $\mathrm{IgD}^{+} \mathrm{B}$ cells in vitro, ${ }^{28}$ and surface expression of BCMA by in vitro generated plasma blasts renders them sensitive to agonist effects of both APRIL and BLyS as indicated by their survival and immunoglobulin secretion. ${ }^{29}$ Accordingly, APRIL could potentiate and/or supplement BLyS activity. On the other hand, TACI (which is bound by both BLyS and APRIL) can transmit to the B cell a negative signal that is vital for the prevention of B cell based autoimmunity. ${ }^{30}$ Accordingly, APRIL could serve as a homoeostatic down modulator of B cell hyperactivity.

Given the potential for APRIL to up modulate or down modulate the "autoimmunogenic" effects of BLyS, it stands to reason that changes in circulating APRIL levels over time could have important ramifications for serological and/or clinical autoimmunity in patients with SLE. We previously characterised BLyS overexpression in a cohort of 68 patients with SLE followed up longitudinally over 12 months. ${ }^{15}$ In this report, we examine APRIL expression in these patients.

\footnotetext{
Abbreviations: APRIL, a proliferation-inducing ligand; BLyS, B lymphocyte stimulator; ELISA, enzyme linked immunosorbent assay; IFN, interferon; SLE, systemic lupus erythematosus; SLEDAI, SLE Disease Activity Index; TNF, tumour necrosis factor
} 

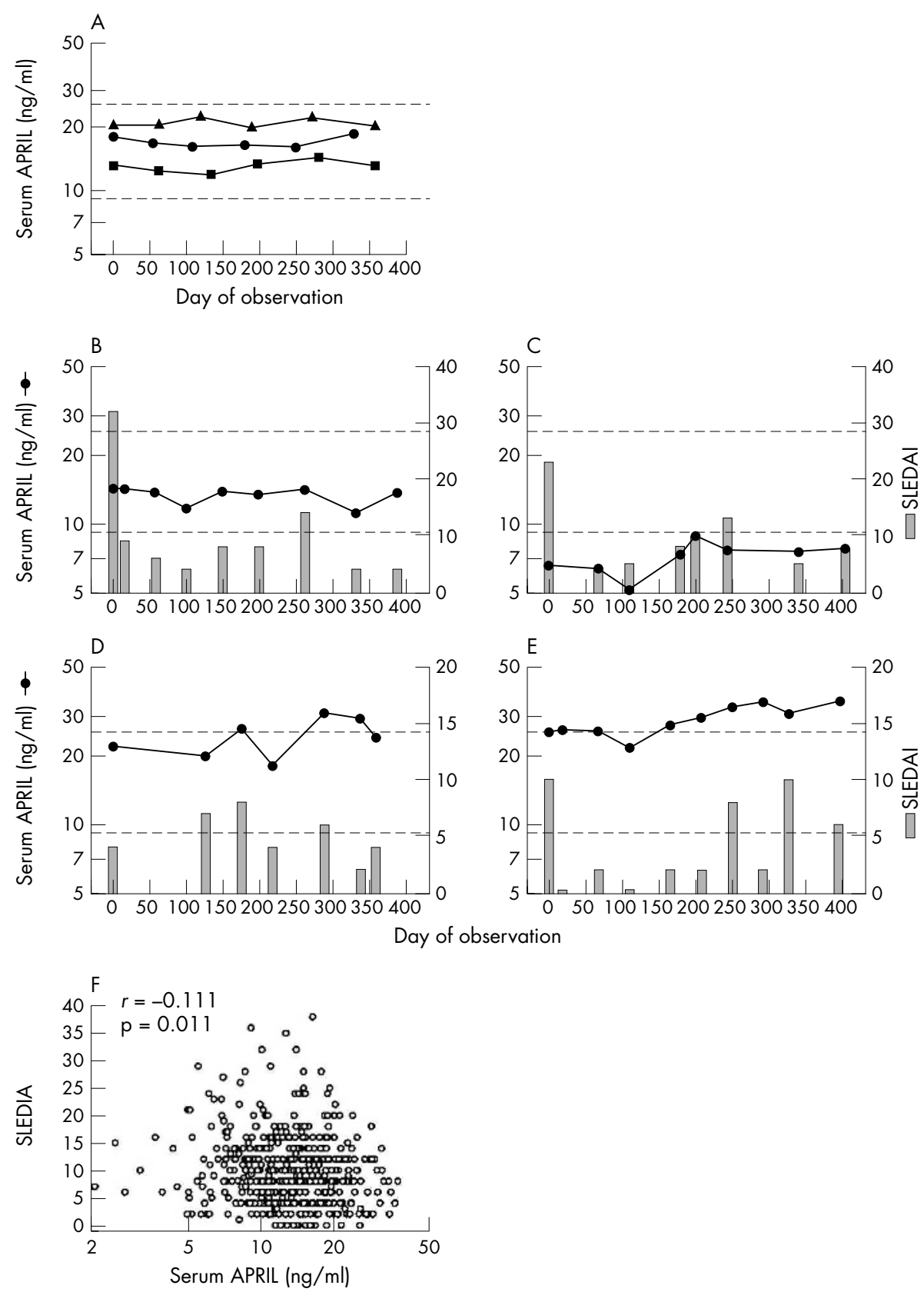

Figure 1 Heterogeneity in serum APRIL phenotypes among patients with SLE and relation to disease activity. (A) Serum samples collected from three representative normal controls (circles, triangles, squares) were assayed for APRIL levels. By definition, the first serum samples were collected on day 0. The dashed lines indicate 2SD above and below the geometric mean of all the values $(n=86)$ obtained from the 15 control subjects and arbitrarily define the "normal" range. (B-E) Serum samples collected from individual patients with SLE were assayed for APRIL levels (circles). Disease activity was measured by SLEDAI (bars). Results for representative patients exhibiting the "persistently normal" serum APRIL phenotype (B), the "persistently low" serum APRIL phenotype (C), the "intermittently raised" serum APRIL phenotype (D), or the "persistently raised" serum APRIL phenotype (E) are illustrated. (F) Serum APRIL levels plotted against the corresponding SLEDAI scores for the 523 individual serum samples from the 68 patients with SLE for whom serum APRIL levels and SLEDAI scores were both determined.

\section{PATIENTS AND METHODS}

\section{Subjects}

The selection criteria, characteristics, and medical management of the 68 patients with SLE and 15 healthy control volunteers who served as the subjects of this report have been previously detailed. ${ }^{15}$ Clinical disease activity was assessed by SLEDAI (SLE Disease Activity Index). ${ }^{31}$ Drugs being taken were recorded, and venous blood samples were collected for laboratory based studies (see below). Doses of corticosteroids other than prednisone were converted to and reported as daily prednisone equivalent dosages. Each of the patients with SLE who were longitudinally studied for 147-420 (median 369) days was seen 3-12 (median 8) times. Day 0 was defined as the day that a given recruited patient or control underwent his/her first clinical and/or laboratory evaluation.

\section{Serum BLyS determination}

Serum BLyS levels for the patients and controls were determined by enzyme linked immunosorbent assay $(\text { ELISA) })^{1432}$ and have recently been reported. ${ }^{15}$ 


\section{Serum APRIL determination}

Serum APRIL levels were determined by ELISA. ${ }^{33}$ To eliminate day to day interassay variability, all serum samples from a given subject were stored at $-70^{\circ} \mathrm{C}$ until the subject's entire observation period was complete. All serum samples collected over the entire observation period from a given subject were assayed on the same day.

The "normal" range for serum APRIL levels was defined as the geometric mean of all the values $(n=86) \pm 2$ SD obtained over time from the 15 control subjects. Each patient with SLE was assigned a serum APRIL phenotype based on his/her serum APRIL levels over time. A "persistently raised" serum APRIL phenotype was assigned when all or all but one of the patient's serum APRIL levels were greater than the upper limit of normal. An "intermittently raised" serum APRIL phenotype was assigned when at least two of the patient's serum APRIL levels were greater than the upper limit of normal and at least two were in the normal range. A "persistently normal" serum APRIL phenotype was assigned when no more than one of the patient's serum APRIL levels was greater than the upper limit of normal. A "persistently low" serum APRIL phenotype was assigned when all or all but one of the patient's serum APRIL levels were less than the lower limit of normal.
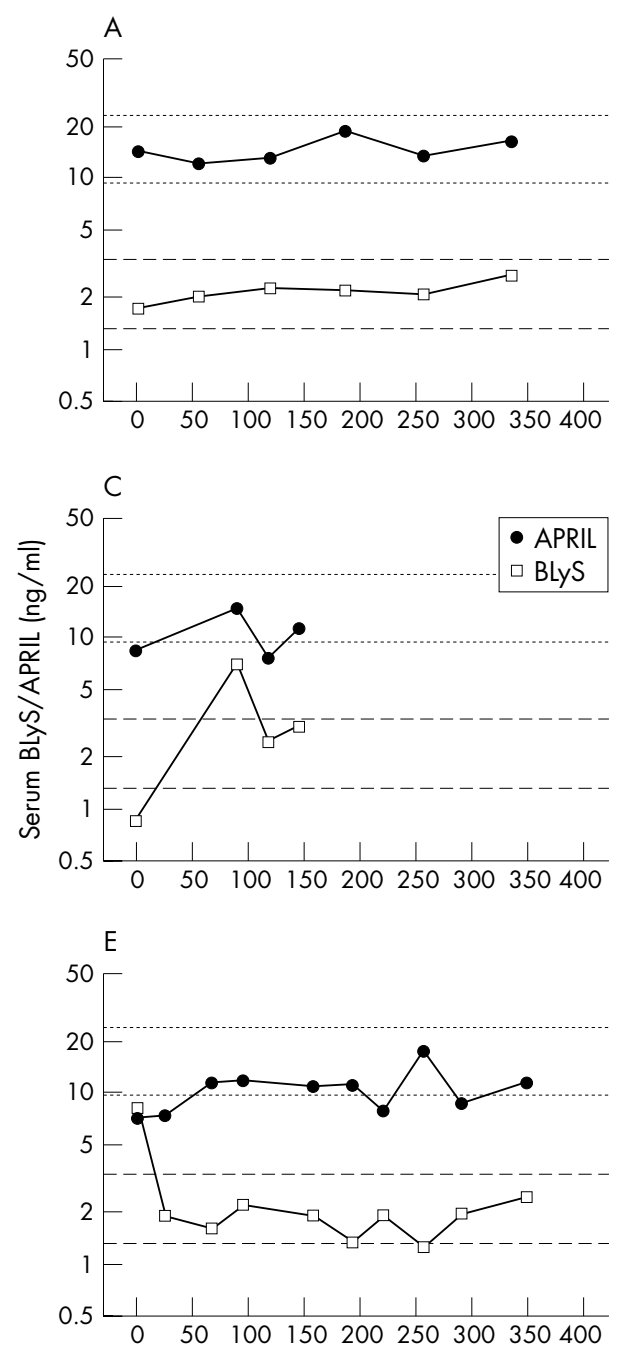

Day of observation

\section{Blood BLyS mRNA determination}

Levels of BLyS mRNA in whole blood were determined by real time quantitative polymerase chain reaction and have recently been reported..$^{15}$

\section{Blood APRIL mRNA determination}

Levels of APRIL mRNA were determined by real time quantitative polymerase chain reaction in a manner identical to that used for BLyS mRNA determination, using the oligonucleotide 5'-AAGCAGCACTCTGTCCTGCACCTGGTT-3' as probe and the primers $5^{\prime}$-GCAGTGCTCACCCAAAAACA-3' and 5'-CCTTGGAGGTGGCGTTAATG-3'. The APRIL amplicon was designed to span the region containing nucleotides 313385 of the APRIL coding sequence. The comparative Delta Ct method (Perkin-Elmer user bulletin No 2 1997) was used for measurement of mRNA using an 18 S ribosomal RNA probe as the endogenous reference. As with serum APRIL determination, all RNA samples from a given subject were stored at $-70^{\circ} \mathrm{C}$ until the subject's entire observation period was complete, at which time all the collected samples for that subject were processed in parallel. Results are presented as APRIL mRNA: $18 \mathrm{~S}$ mRNA ratios.

The "normal" range for blood APRIL mRNA levels and assignment of blood APRIL mRNA phenotypes were
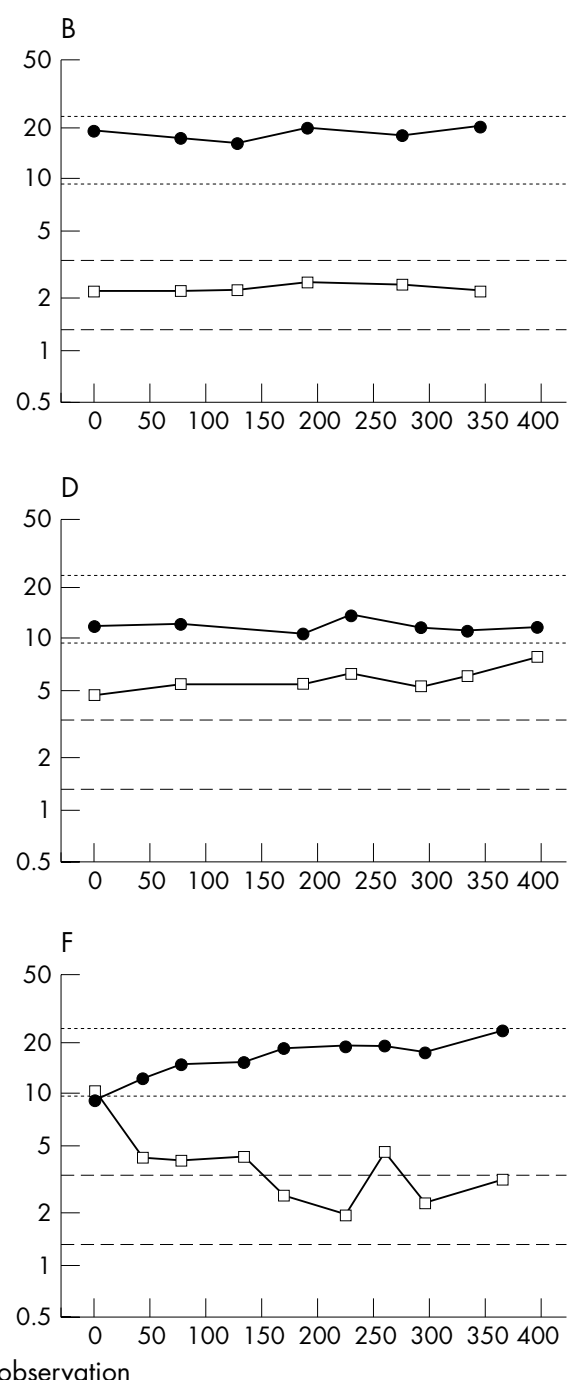

Figure 2 Relation between serum APRIL and serum BLyS levels. Serum samples collected from two representative normal controls (A, B) and four representative patients with SLE (C-F) were assayed for APRIL and BLyS levels. The dotted lines delineate the normal range for serum APRIL, and the dashed lines delineate the normal range for serum BLyS. ${ }^{15}$ 


\begin{tabular}{llllll}
\hline \multicolumn{5}{l}{ Table 1} & APRIL protein and mRNA phenotypes \\
\hline Category & Subjects & Normal & Raised & Intermittent & Low \\
\hline APRIL protein & Controls & $14(93)$ & $1(7)$ & $0(0)$ & $0(0)$ \\
& SLE & $56(82)$ & $2(3)$ & $4(6)$ & $6(9)$ \\
APRIL mRNA & Controls & $15(100)$ & $0(0)$ & $0(0)$ & $0(0)$ \\
& SLE & $48(71)$ & $6(9)$ & $13(19)$ & $1(1)$ \\
\hline \multicolumn{7}{l}{ The values indicate the number (percentage) of subjects with the indicated phenotypes. "Normal" denotes } \\
"persistently normal". "Raised" denotes "persistently raised". "Intermittent" denotes "intermittently raised". \\
"Low" denotes "persistently low". \\
\hline
\end{tabular}

determined in a manner analogous to that described above for serum APRIL.

\section{Statistical analysis}

All analyses were performed using SigmaStat software (SPSS, Chicago, IL). Serum BLyS and serum APRIL levels were $\log$ transformed to achieve normality, permitting parametric testing. Even after log transformation, blood BLYS and APRIL mRNA levels were not normally distributed, so non-parametric testing was performed. Parametric testing between two matched groups was performed by the paired $t$ test. Nominal data were analysed by $\chi^{2}$ analysis of contingency tables or by the Fisher exact test. Correlations were determined by Spearman rank order correlation for interval data that did not display normality and for ordinal data.

\section{RESULTS}

Serum APRIL levels and relation to serum BLyS levels Among normal healthy controls, serum APRIL levels fell within about a threefold range and remained stable over time for any individual subject (fig 1A). This narrow range and stability of serum APRIL levels among normal subjects qualitatively paralleled that of serum BLyS levels, but APRIL levels routinely exceeded those of BLyS by 5- to 10fold (figs 2A and B). All but one of the control subjects exhibited a "persistently normal" serum APRIL phenotype, with the remaining control subject having a "persistently raised" serum APRIL phenotype (table 1).

Among patients with SLE, there was some heterogeneity in the serum APRIL phenotype, with "persistently normal", "persistently low", "intermittently raised", and "persistently raised" phenotypes all being represented (figs lB-E). However, the distribution of serum APRIL phenotypes among patients with SLE did not significantly differ from that

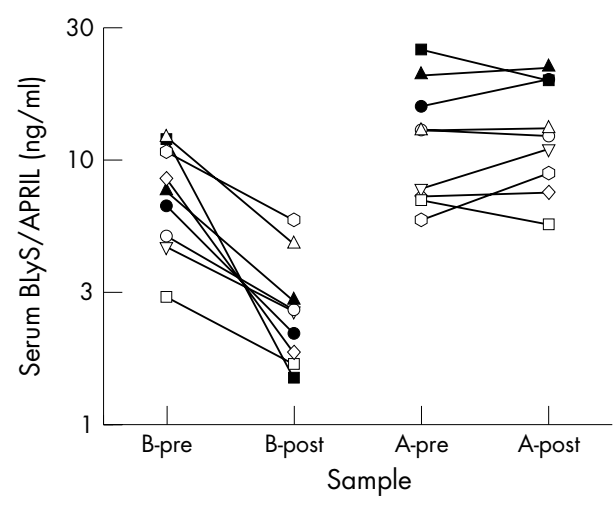

Figure 3 Insensitivity of serum APRIL levels to treatment with high dose corticosteroids. Nine patients with SLE were treated with short courses of high dose corticosteroids. ${ }^{15}$ BLyS levels in pretreatment (B-pre) and posttreatment (B-post) serum samples and APRIL levels in pretreatment (A-pre) and post-treatment (A-post) sera are plotted. among the normal controls $(\mathrm{p}=0.415$, table 1$)$. In aggregate, APRIL levels were raised in only 28 (5.3\%) of the 529 individual serum samples collected from the 68 patients with SLE in comparison with $5(5.8 \%)$ of the 86 individual sera collected from the 15 control subjects $(p=0.953)$. These "normal" results for serum APRIL levels in the patients with SLE are in sharp contrast with the common increase in serum BLyS levels in these same patients. ${ }^{15}$

As a rule, there was non-concordance in the patients with SLE between serum APRIL levels and serum BLyS levels. Although serum levels of BLyS and APRIL paralleled each other to a considerable degree in a minority of patients with SLE (figs 2C and D), changes in serum BLyS levels were discordant with those in serum APRIL levels in the majority of patients (figs 2E and F). This resulted in a lack of overall correlation between serum levels of APRIL and BLyS (data not shown). Further highlighting the discordance between serum APRIL levels and serum BLyS levels are the responses in the nine patients with SLE treated with short courses of high dose corticosteroids. ${ }^{15}$ Whereas the decline in serum BLyS levels was dramatic (geometric mean change from 6.9 to $2.6 \mathrm{ng} / \mathrm{ml}(62 \%$ decrease), $\mathrm{p}<0.001)$, serum APRIL levels were not appreciably affected in these same patients (geometric mean change from 11.1 to $11.9 \mathrm{ng} / \mathrm{ml}(-7 \%$ decrease), $\mathrm{p}=0.412$; fig 3 ).

\section{Blood APRIL mRNA levels and relation to blood BLyS mRNA levels}

The serological results suggested that dysregulation of APRIL among patients with SLE was, at most, modest. To independently assess APRIL expression in patients with SLE by non-serological means, peripheral blood APRIL mRNA levels were measured. In normal controls, blood APRIL mRNA levels showed somewhat greater variations over time, with a broader range of values in comparison with those seen for serum APRIL levels (compare fig 4A with fig lA). None of the 88 APRIL mRNA determinations in control blood samples were raised, and all control subjects exhibited a "persistently normal" blood APRIL mRNA phenotype (table 1).

In the patients with SLE, blood APRIL mRNA levels were sometimes raised despite normal serum APRIL levels (figs 4B and C), although blood APRIL mRNA levels were persistently normal in the majority of patients (figs 4D and E). Of the 526 individual SLE blood samples assayed for APRIL mRNA, 92 $(17.5 \%)$ had raised levels $(\mathrm{p}<0.001$ compared with normal controls). This is reflected in the moderate prevalence of "intermittently raised" and "persistently raised" blood APRIL mRNA phenotypes among patients with SLE $(p=0.018$ compared with normal controls; table 1).

In contrast with the frequent discordance between serum levels of APRIL and BLyS in patients with SLE (fig 2), there routinely was a strong concordance between blood levels of APRIL and BLyS mRNA not just in normal controls (figs 5A and B) but in patients with SLE as well (figs 5C and D). This resulted in a very strong and highly significant correlation 

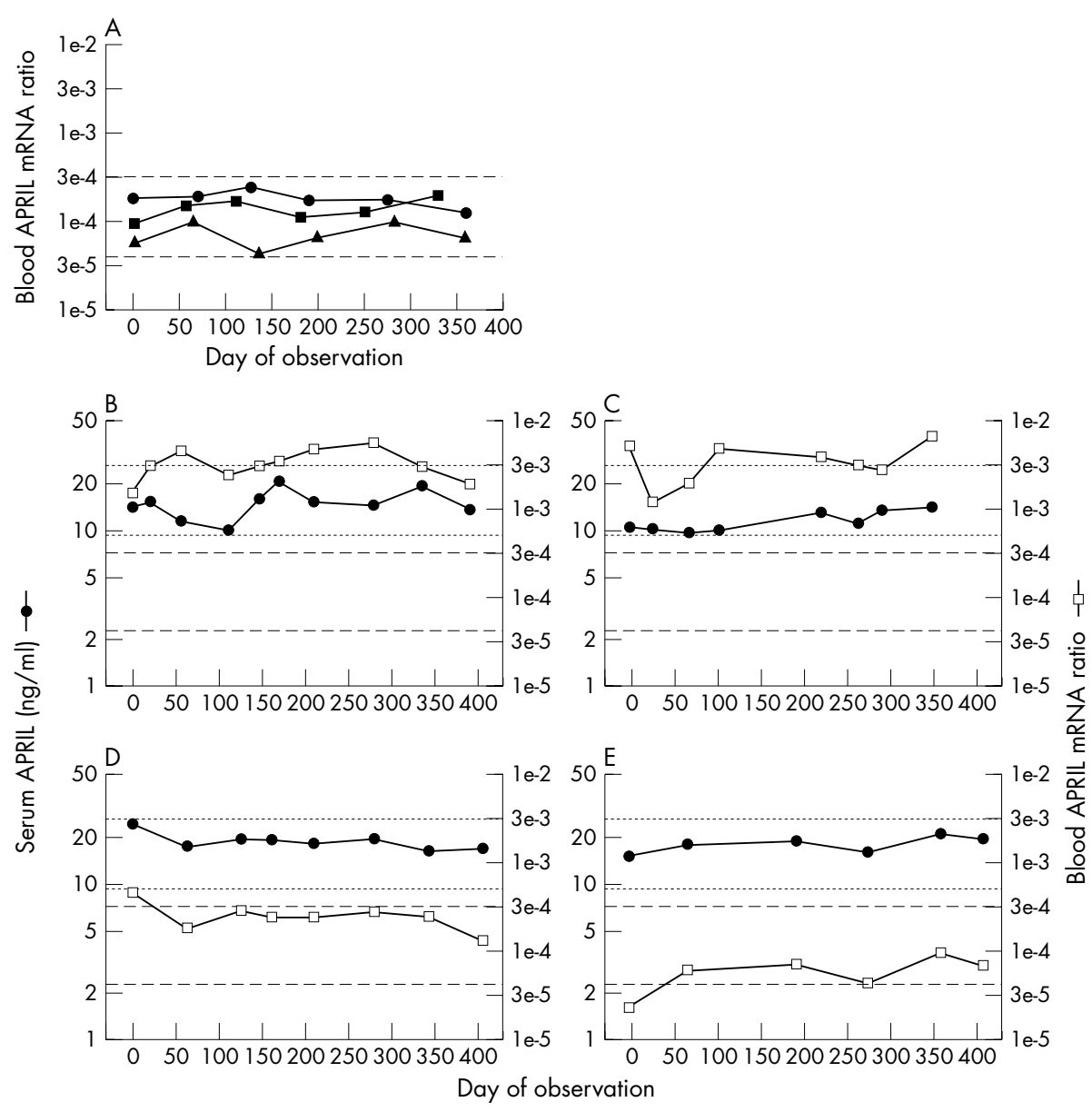

Figure 4 Heterogeneity in blood APRIL mRNA phenotypes among patients with SLE. (A) Peripheral blood samples collected from the same three representative normal controls illustrated in fig $1 \mathrm{~A}$ (circles, triangles, squares) were assayed for APRIL mRNA levels. By definition, the first blood samples were collected on day 0 . The dashed lines indicate 2SD above and below the geometric mean of all the values $(n=88)$ obtained from the 15 control subjects and arbitrarily define the "normal" range. (B-E) Serum APRIL levels (filled circles) and blood APRIL mRNA levels (open squares) were measured. The dotted lines delineate the normal range for serum APRIL, and the dashed lines delineate the normal range for blood APRIL mRNA. In four patients, blood APRIL mRNA levels were markedly raised despite normal serum APRIL levels (B, C). In most patients, blood APRIL mRNA levels remained normal throughout the entire observation periods $(D, E)$.

between blood APRIL mRNA levels and blood BLyS mRNA levels when taking all the SLE blood samples into account (fig 5E).

\section{Inverse correlation between serum APRIL levels and serum anti-dsDNA titres or disease activity}

Although circulating BLyS levels do not correlate well with disease activity in individual patients with SLE, ${ }^{15}$ they do correlate with disease activity and with circulating antidsDNA levels among patients with SLE analysed in aggregate. ${ }^{13-15} 34$ Thus, the prevalent discordance between serum BLyS levels and serum APRIL levels (fig 2) raises the possibility of an inverse relation between serum APRIL levels and BLyS driven biological and/or clinical sequelae.

This was indeed the case. Thirty four patients with SLE harboured a serum anti-dsDNA titre of $\geqslant 1 / 20$ on $\geqslant 2$ observational time points. Although serum APRIL levels and serum anti-dsDNA titres substantially paralleled each other in 10 of these patients (figs 6A and B), an anti-parallel relation was found in the remaining 24 patients (figs 6C and D). When serum samples from all times from all patients were considered, a modest but significant inverse correlation between serum APRIL levels and serum anti-dsDNA titres was found (fig 6E).
A similar inverse correlation was found between serum APRIL levels and disease activity (as measured by SLEDAI). Although serum APRIL levels did not overtly correlate with disease activity over time in individual patients with SLE regardless of their serum APRIL phenotype (figs lB-E), disease activity was modestly, but significantly, inversely correlated with serum APRIL levels when all patient serum samples at all times were collectively analysed (fig IF). No significant correlations between blood APRIL mRNA levels and disease activity or serum anti-dsDNA titres were found (data not shown).

\section{DISCUSSION}

Little information has hitherto been available about circulating APRIL levels in patients with SLE. We now demonstrate that in comparison with the great stability of serum APRIL levels over time in normal donors, serum APRIL levels manifest a limited degree of fluctuation over time in patients with SLE (figs IA-E). This fluctuation in serum APRIL levels in patients with SLE largely occurs within the normal range and results in a spectrum of serum APRIL phenotypes that is not significantly different from that in normal subjects (table 1). Nevertheless, two facets of the APRIL response are striking. 

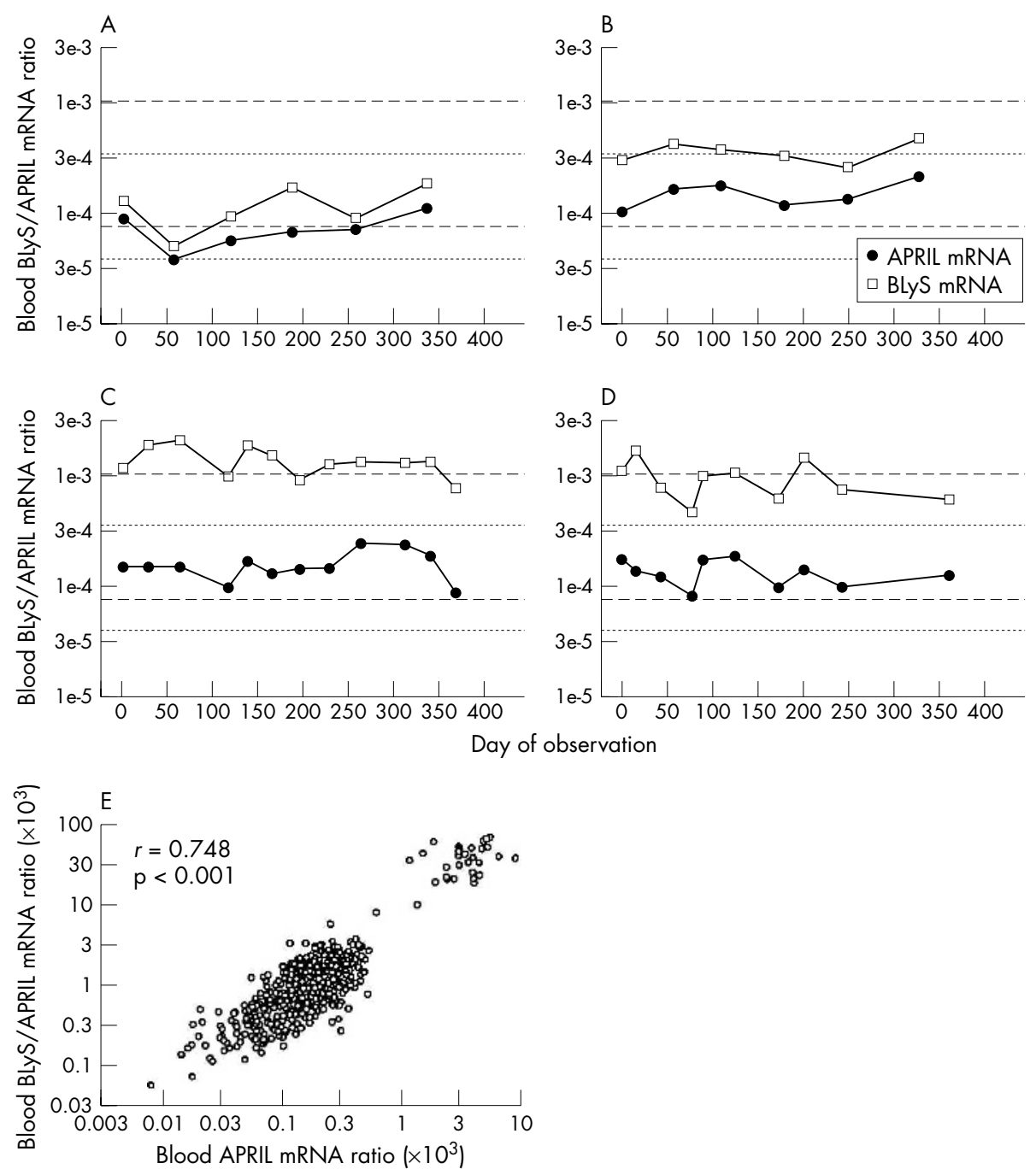

Figure 5 Relation between blood APRIL mRNA levels and blood BLyS mRNA levels. (A-D) Blood collected from two representative normal controls (A, $B$ ) and two representative patients with SLE (C, D) were assayed for APRIL mRNA and BLyS mRNA. The dotted lines delineate the normal range for blood APRIL mRNA, and the dashed lines delineate the normal range for blood BLyS mRNA. ${ }^{15}$ (E) Blood BLyS mRNA levels plotted against blood APRIL mRNA levels for the 526 individual blood samples obtained from the 68 patients with SLE for whom both blood APRIL and BLyS mRNA levels were determined.

Firstly, the usual non-concordance between serum levels of APRIL and BLyS (figs $2 \mathrm{E}$ and F), highlighted by the insensitivity of serum APRIL levels to short courses of high dose corticosteroids despite the exquisite sensitivity of serum BLyS levels to such treatment (fig 3), strongly suggests that APRIL and BLyS are differentially regulated. Although in vitro treatment of dendritic cells (and, probably, other myeloid lineage cells) with interferon (IFN) $\alpha$, IFN $\gamma$, or CD40 ligand (CD40L) results in up regulation of both APRIL and BLyS, ${ }^{28}$ and although blood APRIL and BLyS mRNA levels did strongly correlate with each other in the patients with SLE (fig 5), APRIL is expressed in vivo by other cell types, including cells from prostate, colon, and pancreas. ${ }^{19}$ It is entirely plausible that the bulk of APRIL produced in vivo arises from sources other than myeloid lineage cells. The "non-myeloid APRIL" may be regulated in a manner different from that of "myeloid APRIL". Alternatively, it is possible that factors other than IFN $\alpha$, IFN $\gamma$, or CD40L are major post-transcriptional regulators of APRIL and/or BLyS in vivo. These as yet unidentified factors may have differential effects on APRIL and BLyS. Either possibility could explain why the degree of dysregulation in patients with SLE of APRIL is quantitatively much more limited than that of BLyS (compare table 1 with our previous study ${ }^{15}$ ).

Secondly, changes in serum APRIL levels in patients with SLE, despite often remaining in the "normal" range, appear to be biologically and clinically important. APRIL binds to $\mathrm{TACI}^{20-23}$ which (presumably) delivers a negative signal to the B cell. ${ }^{30}$ APRIL, however, does not bind to BAFFR, so it does not deliver a BAFFR mediated positive signal to the $\mathrm{B}$ cell. ${ }^{162425} 27$ Because circulating levels of APRIL are considerably greater than those of BLyS (fig 2), even small changes in serum APRIL levels may have substantial ramifications for B cell biology. Serum APRIL levels and serum anti-dsDNA titres usually demonstrated an anti-parallel relation (figs 6C and D), and a modest inverse correlation between the two was found when all samples from all the anti-dsDNA positive patients were considered (fig 6E). Importantly, despite serum APRIL levels not overtly correlating with disease activity over time for any individual patient with SLE (figs 1B-E), a modest inverse correlation was seen when all patients at all times were considered (fig lF). Although purely speculative, it may be that the polymorphism of the april gene associated 

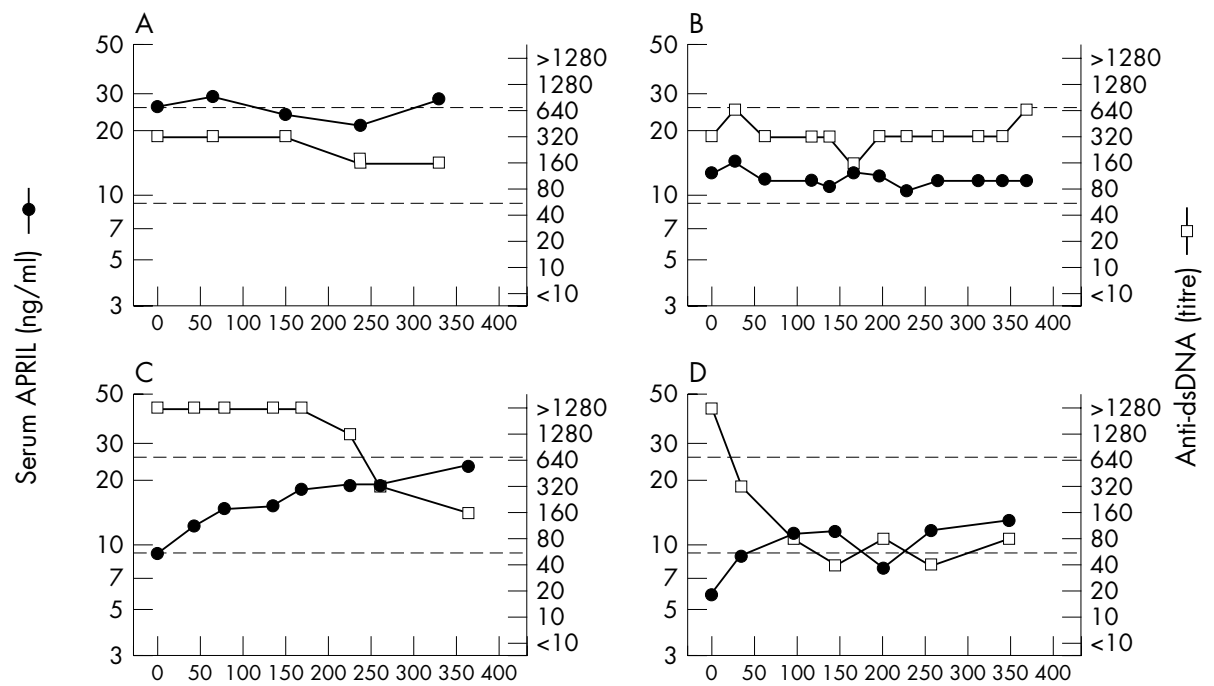

Day of observation

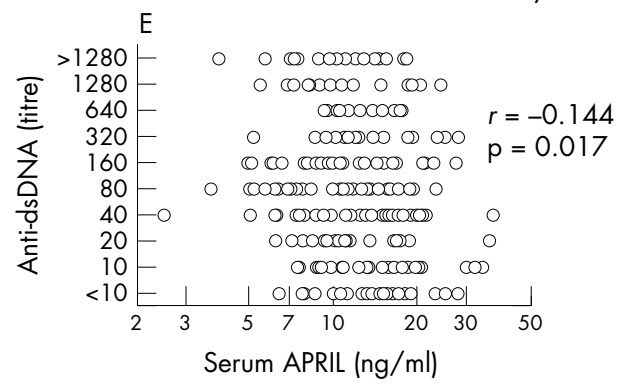

Figure 6 Relation between serum APRIL levels and serum anti-dsDNA titres. (A, B) Serum APRIL levels (filled circles) and serum anti-dsDNA titres (open squares) were determined in two representative patients with SLE in whom changes in serum APRIL levels largely paralleled those in serum antidsDNA titres. (C, D) Serum APRIL levels and serum anti-dsDNA titres were determined in two representative patients with SLE in whom changes in serum APRIL levels were largely anti-parallel to those in serum anti-dsDNA titres. (E) Serum anti-dsDNA titres plotted against serum APRIL levels for the 275 individual serum samples obtained from the 34 anti-dsDNA positive patients with SLE.

with human SLE ${ }^{35}$ imparts upon APRIL a reduced biological half life and/or decreased binding affinity to TACI, either of which would reduce the negative regulatory input to B cells. The fact that the inverse correlations between serum APRIL levels and serum anti-dsDNA titres or disease activity are only modest demonstrates that the precise role for APRIL in regulation of autoantibody production and/or disease activity requires further elucidation.

Circulating soluble APRIL may not be the only form of APRIL relevant to B cell biology and/or B cell based autoimmunity. A biologically active, naturally occurring hybrid protein composed of the cytoplasmic and transmembrane domains of TWEAK (another member of the TNF ligand superfamily) fused to the APRIL C-terminal domain is expressed by T cells and monocytes. ${ }^{36}$ What regulatory role, if any, this cell surface APRIL hybrid plays in vivo remains speculative. There is no a priori reason to exclude the possibility of the APRIL moiety interacting with B cell surface TACI, ${ }^{20-23}$ which could transmit a negative signal to the $\mathrm{B}$ cell, $^{30}$ and/or with B cell surface BCMA, ${ }^{20} 2123$ which could transmit a positive signal to plasma blasts. ${ }^{29}$ In addition, alternate splice forms of APRIL have been identified (37 and HGS, unpublished observations). Whether these alternate splice forms result in variants of APRIL and what, if any, biological activities these putative APRIL variants have, remains to be determined. Further investigation into these issues is warranted.

The apparent down regulatory property of APRIL may have important ramifications for BLyS targeted treatment in SLE. It is tempting to speculate that agents which can neutralise only BLyS while having no effect on APRIL may be preferable to those agents which can neutralise both BLyS and APRIL. Ultimately, clinical experience will determine which BLyS antagonists are most efficacious in the treatment of SLE and other B cell based autoimmune disorders.

\section{ACKNOWLEDGEMENTS}

The authors thank Dong Xu for handling and cataloguing the patient samples and all the subjects for their participation in the study. This work was supported in part by grants from the NIH (AR41006) and the Alliance for Lupus Research and a gift from Lupus International.

\section{Authors' affiliations}

W Stohl, S Metyas, S-M Tan, G S Cheema, B Oamar, Division of Rheumatology, Department of Medicine, Los Angeles County + University of Southern California Medical Center and University of Southern California Keck School of Medicine, Los Angeles, CA 90033, USA

V Roschke, Y Wu, K P Baker, D M Hilbert, Human Genome Sciences, Inc, Rockville, MD 20850, USA

\section{REFERENCES}

1 Moore PA, Belvedere O, Orr A, Pieri K, LaFleur DW, Feng P, et al. BLyS member of the tumor necrosis factor family and $B$ lymphocyte stimulator. Science 1999;285:260-3.

2 Shu H-B, Hu W-H, Johnson H. TALL-1 is a novel member of the TNF family that is down-regulated by mitogens. J Leukocyte Biol 1999;65:680-3.

3 Schneider P, MacKay F, Steiner V, Hofmann K, Bodmer J-L, Holler N, et al. BAFF, a novel ligand of the tumor necrosis factor family, stimulates $B$ cell growth. J Exp Med 1999;189:1747-56. 
4 Mukhopadhyay A, Ni J, Zhai Y, Yu G-L, Aggarwal BB. Identification and characterization of a novel cytokine, THANK, a TNF homologue that activates apoptosis, nuclear factor- $\kappa \mathrm{B}$, and $\mathrm{c}$-Jun $\mathrm{NH}_{2}$-terminal kinase. J Biol Chem 1999:274:15978-81.

5 Tribouley C, Wallroth M, Chan V, Paliard X, Fang E, Lamson G, et al. Characterization of a new member of the TNF family expressed on antigen presenting cells. Biol Chem 1999;380:1443-7.

6 Gross JA, Johnston J, Mudri S, Enselman R, Dillon SR, Madden K, et al. TACl and $\mathrm{BCMA}$ are receptors for a TNF homologue implicated in B-cell autoimmune disease. Nature 2000;404:995-9.

7 Thompson JS, Schneider P, Kalled SL, Wang L, Lefevre EA, Cachero TG, et al. BAFF binds to the tumor necrosis factor receptor-like molecule $B$ cell maturation antigen and is important for maintaining the peripheral $\mathrm{B}$ cell population. J Exp Med 2000;192:129-35.

8 Do RKG, Hatada E, Lee H, Tourigny MR, Hilbert D, Chen-Kiang S. Attenuation of apoptosis underlies B lymphocyte stimulator enhancement of humoral immune response. J Exp Med 2000;192:953-64

9 Batten M, Groom J, Cachero TG, Qian F, Schneider P, Tschopp J, et al. BAFF mediates survival of peripheral immature B lymphocytes. J Exp Med 2000; 192:1453-65.

10 Hsu BL, Harless SM, Lindsley RC, Hilbert DM, Cancro MP. Cutting edge: BLyS enables survival of transitional and mature $B$ cells through distinct mediators. $J$ Immunol 2002;168:5993-6.

11 Mackay F, Woodcock SA, Lawton P, Ambrose C, Baetscher M, Schneider P, et al. Mice transgenic for BAFF develop lymphocytic disorders along with autoimmune manifestations. J Exp Med 1999;190:1697-710.

12 Khare SD, Sarosi I, Xia X-Z, McCabe S, Miner K, Solovyev I, et al. Severe B cell hyperplasia and autoimmune disease in TALL-1 transgenic mice. Proc Natl Acad Sci USA 2000;97:3370-75.

13 Zhang J, Roschke V, Baker KP, Wang Z, Alarcón GS, Fessler BJ, et al. Cutting edge: a role for $B$ lymphocyte stimulator in systemic lupus erythematosus. $\mathrm{J}$ Immunol 2001;166:6-10.

14 Cheema GS, Roschke V, Hilbert DM, Stohl W. Elevated serum B lymphocyte stimulator levels in patients with systemic immune-based rheumatic diseases. Arthritis Rheum 2001;44:1313-19.

15 Stohl W, Metyas S, Tan S-M, Cheema GS, Oamar B, Xu D, et al. B lymphocyte stimulator overexpression in patients with systemic lupus erythematosus: longitudinal observations. Arthritis Rheum 2003;48:3475-86.

16 Kayagaki N, Yan M, Seshasayee D, Wang H, Lee W, French DM, et al. BAFF/BLyS receptor 3 binds the B cell survival factor BAFF ligand through a discrete surface loop and promotes processing of NF-кB2. Immunity 2002; 10:515-24.

17 Baker KP, Edwards BM, Main SH, Choi GH, Wager RE, Halpern WG, et al. Generation and characterization of LymphoStat-B, a human monoclonal antibody that antagonizes the bioactivities of $B$ lymphocyte stimulator. Arthritis Rheum 2003:48:3253-65.

18 Furie R, Stohl W, Ginzler E, Becker M, Mishra N, Chatham W, et al. Safety, pharmacokinetic and pharmacodynamic results of a phase 1 single and double dose-escalation study of Lymphostat- $B$ (human monoclonal antibody to BLyS) in SLE patients [abstract]. Arthritis Rheum 2003;48(suppl):S377.

19 Hahne M, Kataoka T, Schröter M, Hofmann K, Irmler M, Bodmer J-L, et al. APRIL, a new ligand of the tumor necrosis factor family, stimulates tumor cell growth. J Exp Med 1998; 188:1 185-90.

20 Marsters SA, Yan M, Pitti RM, Haas PE, Dixit VM, Ashkenazi A. Interaction of the TNF homologues BLyS and APRIL with the receptor homologues BCMA and TACl. Curr Biol 2000;10:785-8.
21 Yu G, Boone T, Delaney J, Hawkins N, Kelley M, Ramakrishnan M, et al. APRIL and TALL- 1 and receptors BCMA and TACl: system for regulating humoral immunity. Nat Immunol 2000;1:252-6.

22 Wu Y, Bressette D, Carrell JA, Kaufman T, Feng P, Taylor K, et al. Tumor necrosis factor (TNF) receptor superfamily member TACl is a high affinity receptor for TNF family members APRIL and BLyS.J Biol Chem 2000;275:35478-85.

23 Rennert P, Schneider P, Cachero TG, Thompson J, Trabach L, Hertig S, et al. A soluble form of $B$ cell maturation antigen, a receptor for the tumor necrosis factor family member APRIL, inhibits tumor cell growth. J Exp Med 2000;192:1677-83.

24 Thompson JS, Bixler SA, Qian F, Vora K, Scott ML, Cachero TG, et al. BAFF$\mathrm{R}$, a novel TNF receptor that specifically interacts with BAFF. Science 2001;293:2108-11

25 Yan M, Brady JR, Chan B, Lee WP, Hsu B, Harless S, et al. Identification of a novel receptor for $B$ lymphocyte stimulator that is mutated in a mouse strain with severe B cell deficiency. Curr Biol 2001;11:1547-52.

26 Stein JV, López-Fraga M, Elustondo FA, Carvalho-Pinto CE, Rodríguez D, Gómez-Caro R, et al. APRIL modulates B and T cell immunity. J Clin Invest 2002:109:1587-98.

27 Claudio E, Brown K, Park S, Wang H, Siebenlist U. BAFF-induced NEMO-

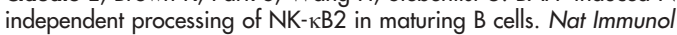
2002;3:958-65.

28 Litinskiy MB, Nardelli B, Hilbert DM, He B, Schaffer A, Casali P, et al. DCs induce CD40-independent immunoglobulin class switching through BLyS and APRIL. Nat Immunol 2002;3:822-9.

29 Avery DT, Kalled SL, Ellyard Jl, Ambrose C, Bixler SA, Thien M, et al. BAFF selectively enhances the survival of plasmablasts generated from human memory B cells. J Clin Invest 2003;1 12:286-97.

30 Seshasayee D, Valdez P, Yan M, Dixit VM, Tumas D, Grewal IS. Loss of TACI causes fatal lymphoproliferation and autoimmunity, establishing $\mathrm{TACl}$ as an inhibitory BLyS receptor. Immunity 2003;18:279-88.

31 Bombardier C, Gladman DD, Urowitz MB, Caron D, Chang CH, Committee on Prognosis Studies in SLE. Derivation of the SLEDAI: a disease activity index for lupus patients. Arthritis Rheum 1992:35:630-40.

32 Nardelli B, Belvedere O, Roschke V, Moore PA, Olsen HS, Migone TS, et al. Synthesis and release of B-lymphocyte stimulator from myeloid cells. Blood 2001; 97: 198-204.

33 Tan S-M, Xu D, Roschke V, Perry JW, Arkfeld DG, Ehresmann GR, et al. Local production of B lymphocyte stimulator protein and APRIL in arthritic joints of patients with inflammatory arthritis. Arthritis Rheum 2003;48:982-92.

34 Petri M, Stohl W, Chatham W, McCune WJ, Butler T, Ryel J, et al. BLyS plasma concentrations correlate with disease activity and levels of anti-dsDNA autoantibodies and immunoglobulins (lg) in a SLE patient observational study [abstract]. Arthritis Rheum 2003;48(suppl):S655.

35 Koyama T, Tsukamoto H, Masumoto K, Himeji D, Hayashi K, Harada M, et al. A novel polymorphism of the human APRIL gene is associated with systemic lupus erythematosus. Rheumatology (Oxford) 2003;42:980-5.

36 Pradet-Balade B, Medema JP, López-Fraga M, Lozano JC, Kolfschoten GM, Picard A, et al. An endogenous hybrid mRNA encodes TWE-PRIL, a functional cell surface TWEAK-APRIL fusion protein. EMBO J 2002;21:5711-20.

37 Craxton A, Magaletti D, Ryan EJ, Clark EA. Macrophage- and dendritic celldependent regulation of human B-cell proliferation requires the TNF family ligand BAFF. Blood 2003;101:4464-71. 